\title{
Centroamérica: democratización y ajuste estructural
}

Gilberto Castañeda Sandoval

CIDE
$\mathrm{E}$

n el último tiempo, en Centroamérica, democratización y ajuste estructural son procesos que muestran una tendencia a la generalización y uniformidad. Al parecer, hay un consenso extendido sobre la necesidad de la democratización -necesidad muy marcada en El Salvador y Guatemala- y el ajuste estructural, diferencias más, diferencias menos, se aplica ya en toda la región bajo el modelo preconizado por el Fondo Monetario Internacional (FMI), el Banco Mundial (BM) y la Agencia Internacional para el Desarrollo de Estados Unidos (AID). Sin embargo, hay razones para poner en duda tal consenso, al tiempo que es necesario señalar que el ajuste ha sido contra- rio a los propósitos democráticos, sobre todo al descargar sus costos principalmente sobre los campesinos, los asalariados del campo y de la ciudad y, en Guatemala, los pueblos indígenas. Éstas son las ideas que intento exponer en este trabajo.

\section{LA CUESTIÓN DEMOCRÁTICA EN CENTROAMÉRICA}

La búsqueda de la democracia tiene raíces históricas profundas en Centroamérica. Sus principales desarrollos del último tiempo datan de los años $\mathbf{4 0}$ de este siglo, cuando fueron derrocadas las dictaduras del istmo, con excepción de la dinastía Somoza, 
que se prolongó hasta 1979. ${ }^{1}$ Pese a ello, ha sido un camino lleno de obstáculos y sumamente sinuoso.

En este momento, las dificultades $y$ retos que enfrenta el logro de la democracia en Centroamérica son tales que campea la relatividad. Por ello es posible afirmar que la misma se expresa en la búsqueda de "alguna modalidad democrática que a la vez pueda constituir el punto de partida para alguna forma de crecimiento con desarrollo". ${ }^{2}$ Es, en efecto, un esfuerzo que por ahora no ofrece desenlaces previsibles, particularmente en Guatemala, El Salvador y Nicaragua. Entre los principales obstáculos que se alzan frente al esfuerzo democratizador se pueden anotar, entre otros, los siguientes:

a) Las dificultades por las que atraviesan actualmente los movimientos 352.

1 Torres Rivas, "Centroamérica", 1989, p.

2 En Guatemala, por ejemplo, en octubre de 1990 fue asesinado Humberto González Gamarra, dirigente del Comité Proformación del Partido Unidad Revolucionara Democrática (URD). En diciembre fue asesinado otro dirigente del mismo comité, Manuel Collado; con este asesinado se decret6 la muerte de la URD, aunque algunos de sus integrantes persisten en sostenerla. Despues, en abril del año siguiente, fue asesinada Dinora Pérez, dirigente de la agrupación Guatemala Unida, un desprendimiento del Partido Socialista Democrático (PSD) que se dio en el contexto de las elecciones generales pasadas, por desacuerdos con la política de alianzas que siguió la dirigencia del partido, la cual participa actualmente en el gobierno de Serrano Elías. Este asesinato y el hostigamiento de los restantes miembros de la agrupación terminó con su desmantelamiento y la salida al exilio de la mayoría de sus principales integrantes. Cabe señalar que todo esto ocurrió a pesar de que Guatemala Unida había participado dentro de las elecciones apoyando a la DC. populares y revolucionarios en el área $y$, en particular, en Guatemala y El Salvador. Con ello y la agudeza de los retos que presentan los procesos político-sociales en el área, se ha abierto paso a la necesidad de buscarle soluciones negociadas a los conflictos como requisito para cualquier intento democrático, pero en un marco en el que la perspectiva no es del todo visible, pese a los avances que al respecto del logro de la paz muestra El Salvador.

b) Las paradojas que plantea esta tendencia a la negociación cuando se da en un marco en el que la violencia estatal no cede terreno, como es el caso de Guatemala. Aquí, el objetivo pareciera ser golpear a aquellos sectores organizados de la población que pueden tener un papel dentro de las negociaciones y, de esta manera; imponer a la guerrilla una suerte de rendición incondicional o, en todo caso, limitar sus posibilidades de acción legal futura, derivadas de un acuerdo de paz.

c) Las dificultades e incógnitas que plantea la experiencia nicaragüense donde el Frente Sandinista sigue siendo para muchos, sobre todo en Estados Unidos, el enemigo a vencer.

d) Los efectos negativos de la crisis económica y del ajuste estructural sobre las mayorías nacionales, debilitando su capacidad de organización y movilización, así como de conquista de triunfos, ya bastante mermada por la represión en casos como el de Guatemala o, simplemente, acarreando pérdidas y cercenamientos de logros ya alcanzados como ocurre en Costa Rica. 
e) El acrecentado peso de Estados Unidos en la región en momentos en que la crisis en Europa del Este y la URSS y las dinámicas de reestructuración económica y política mundiales le dejan mano libre sobre el hemisferio. Adicional a todo ello y ante el empuje de estas dinámicas tan diversas y complejas, muchas veces contradictorias, Centroamérica está cambiando aceleradamente. Sin embargo, esto ocurre -ivaya paradoja!- sin remover a fondo el viejo sistema económicosocial excluyente y concentrador. ${ }^{3}$

\section{Centroamérica cambia deNTRo DE SUS VIEIOS PATRONES}

En Centroamérica, el eje de acumulación agroexportador está siendo desplazado -aunque no sustituido- por las llamadas exportaciones no tradicionales y los ingresos provenientes de las remesas familiares del exterior (los costos más relevantes son los de El Salvador y Guatemala). A ellos se suman los negocios relacionados con el narcotráfico (particularmente en Costa Rica, Guatemala y Honduras), al tiempo que ganan terreno las inversiones especulativas. ${ }^{4}$ 1983.

3 Véase Mayorga Quirós, "Crecimiento",

Por ejemplo, en Guatemala las llamadas exportaciones tradicionales (café, algodón, azúcar, banano, carne y cardamomo) conservan un peso significativo en las exportaciones totales y, aunque tienden a la baja, esto no es todavía definitivo. En 1980, las exportaciones tradicionales de Guatemala representaron el $64.4 \%$, en 1986 el $67.4 \%$ y en 1989 el $55.7 \%$, pero lo más significativio es que, dentro de estos porcentajes, continúa siendo notorio el peso del café: $30.5,48.1$
En este contexto; se dan cambios importantes en la composición y estratificación social. Esto es claramente visible con el desplazamiento de importantes sectores de la población hacia las llamadas economías "informal" $y$ "subterránea" (narcotráfico, vicio, corrupción, etc.), al tiempo que disminuye el peso de los obreros, aumenta el desempleo y el Estado reduce su tamaño al disminuir, principalmente, la inversión social, aunque se mantiene, y en ocasiones se incrementa, el gasto policial 'y militar. ${ }^{5}$ A esto se suman las migraciones internacionales de fuerza de trabajo -particularmente hacia Estados Unidos- y la persistencia de los refugiados internos y externos, asi como de los exiliados (particularmente en los casos de El Salvador y Guatemala), cuyos flujos no cesan del todo. Finalmente, se da el incremento incesante de la pobreza. ${ }^{6}$

y $33.2 \%$ respectivamente, para los mismos anos, Peña, Ajuste, 1991.

5 La magniud de la pobreza en Centroamérica aumentó sensiblemente entre 1980-1985. El porcentaje de pobreza pasó del $61 \%$ en 1980 al $75 \%$ en 1985 , mientras que la pobreza extrema pas6 del $39 \%$ al $51 \%$ en el mismo periodo. Por países, los rangos de la pobreza y pobreza extrema para 1985 son, respectivamente: El Salvador $87 \%$ y $51 \%$, Guatemala $83 \%$ y $64 \%$, Honduras $79 \%$ y $57 \%$, Nicaragua $69 \%$ y $38 \%$ y Costa Rica $28 \%$ y $16 \%$. CEPAL, Centroamérica, 1991, cuadro 1, p. 2.

6 En Honduras, por ejemplo, una huelga bananera de importantes dimensiones que estallo en torno a demandas salariales y laborales a principios de 1990, fue violentamente reprimida. Para varios analistas hondureños este suceso, desconocido en los últimos tiempos, es un signo ominoso para el futuro. En Guatemala y El Salvador sobran los ejemplos, pero es más significativo que ni siquiera el sistema costarricense haya podido escapar a la tónica dominante. Cito 
Mientras tanto, la relación Estadosociedad civil conserva sus rasgos autoritarios esenciales. Es más, ante el descontento y la protesta, muchos de ellos se profundizan y amplian de diversas y nuevas maneras. Las organizaciones sindicales y populares, por ejemplo, siguen bajo persecución y no surge un empresariado dinámico con un proyecto "moderno". Desde luego, cualquier posición de corte revolucionario está proscrita. Así, prácticamente, el único proyecto en curso es el del ajuste neoliberal. En este contexto el consenso democratizador resulta aparente.

\section{¿DE QUÉ DEMOCRACIA SE TRATA?}

Para las mayorías nacionales en Centroamérica, la democracia es imposi-

como ejemplo la protesta del 22 de julio de 1991 donde un "numeroso grupo de vendedores reaccionó con violencia contra el edificio de la municipalidad de San José [la ciudad capital], luego de que el Ministerio de Obras Públicas y Transportes inició la eliminación de los puestos de venta del centro de la ciudad. Los ventanales del edificio municipal fueron destruidos y varios negocios saqueados". El caso ejemplifica no sólo la tendencia al uso de métodos de coerción física para resolver problemas generados por la crisis y la respuesta violenta de los afectados sino que, además, refleja el tipo de contradicciones que genera la propia política de ajuste. "El comercio ambulante es la actividad predominante en el llamado 'sector informal urbano' $y$ con el paso del tiempo probablemente se convertirá en el refugio permanente de miles de trabajadores sin empleo", de la burocracia cesante, por ejemplo, y "las políticas de ajuste introducidas en Costa Rica están convirtiendo al comercio callejero o a la delincuencia en las opciones de sobrevivencia que tienen los trabajadores que no encuentran empleo". Morales G., "Costa Rica", 1991, pp. 15. 16. ble mientras subsistan el terror estatal y el carácter excluyente y concentrador del sistema económico-social. Para los sectores conservadores de Estados Unidos y Centroamérica, y aun para algunos otros a nivel internacional, ello no es asi. Para tales sectores, la democratización es posible si tan solo se dan elecciones limpias y verificables; el contexto en que ocurran no es importante. Este puede ser una guerra de agresión (Nicaragua), la persecución y exclusión sistemática de los opositores (El Salvador y Guatemala), una invasión armada (Panamá).

Desde luego, la apertura electoral es importante para la democratizaciớn, pero no es suficiente. Más importante, como punto de partida para tal democratización sería la vigencia efectiva del Estado de derecho. Pero sobre todo, se necesita superar los limites que impone a la democracia la estructura económico-social dominante. Esto es particularmente importante ahora en relación con los límites y obstáculos derivados del ajuste estructural. Los mismos afectan aun a sociedades como la costarricense que, desde 1948, viene acumulando experiencias básicamente democráticas en el marco de importantes logros sociales hoy vulnerables o con riesgo de perderse.

Como se sabe, el ajuste estructural impulsa la apertura externa de la economía al privilegiar las exportaciones. Para ello plantea elevar la competitividad mediante la eliminación de las barreras arancelarias y la aplicación de una política cambiaria "realista", es decir, que obedezca las "señales" del mercado internacional. Además, hay que eliminar el déficit presupuestario 
reduciendo el tamaño y los gastos del Estado, cuestión que se aplica en lo fundamental a la inversión social. El objetivo clave es crecer para pagar la deuda bajo el supuesto de que así se sentarán bases de confianza para que llegue la inversión extranjera. Desde luego, en un contexto como el de Centroamérica, tales medidas -uniformes para todos los países deudores- resultan una bomba de tiempo. Hay ya algunos indicios a la vista. ${ }^{7}$

7 "En realidad, las supuestas 'ventajes comparativas' son cada vez más difíciles de discernir, ya que en materia de producción y eficiencia se basan, con creciente intensidad, en contradicciones adquiridas y, cada vez menos, en factores productivos naturales", es decir en las llamadas ventajas estáticas que "existen en pocos casos". Respecto de las ventajas dinámicas

\section{LOS EFECTOS NEGATTVOS DEL AJUSTE}

En las condiciones de la mayoría de los países centroamericanos, con unas fuerzas productivas severamente castigadas por la crisis, el conflicto y el atraso, la búsqueda de competitividad se da -dentro de una óptica extremadamente conservadora de los sectores empresariales- por medio de una ma-

-las adquiridas-, se requiere del "esfuerzo sistemático de la sociedad nacional. La 'riqueza de las naciones' se basa cada vez más en la competitividad internacional de las economías nacionales [...] Las experiencias asiáticas (Japón, Corea del Sur, Taiwán) revelan que la única manera permanente de acrecentar la competitividad consiste en vincular institucionalmente a las empresas mediante sistemas informativos eficientes (de mercado y de asesoramiento tecnologico), fomentar la cooperación con centros

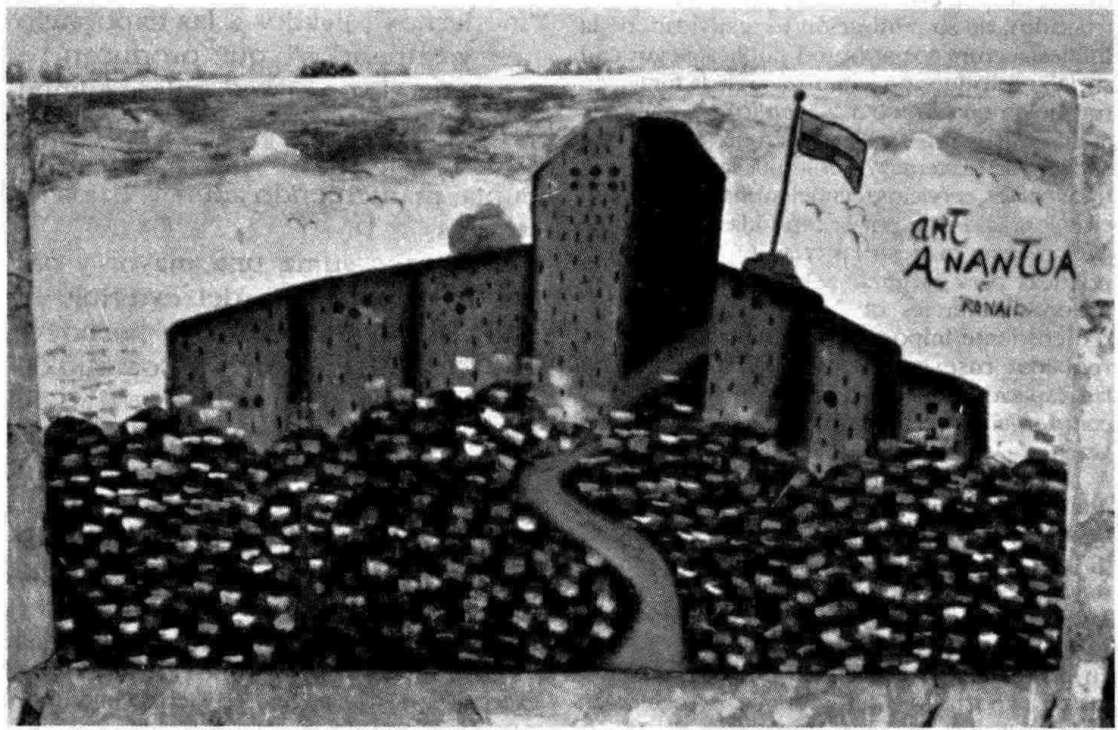


yor compresión de los salarios y con la eliminación creciente de las conquistas laborales y sociales en general. Esto se debe a que, en lo esencial, dicha competitividad resulta dificil de lograr mediante las costosas inversiones que exigiría la incorporación de tecnología de punta. Por ello la tendencia es privilegiar de nueva cuenta las viejas "ventajas comparativas", es decir, la abundancia de recursos naturales y de mano de obra baratos, que son en definitiva las ventajas menos importantes en este momento de acelerada transformación productiva mundial. ${ }^{8}$

En el caso del bajo costo de la fuerza

públicos y privados de investigación aplicada, articular la industria con los servicios y contar con empresas comercializadoras permanentes. El potencial económico depende de la calificación de la fuerza de trabajo (sistema de instrucción), de su motivación y creatividad, de la infraestructura social (salud pública, seguridad social, reproducción garantizada de la fuerza de trabajo en un sentido más amplio), así como de la infraestructura económica en un sentido más restringido (sistemas de comunicación, energía, transportes, servicios tecnocientíficos). También está condicionada por la capacidad del sistema polírico para manejar las crisis [...] Además, las políticas para asegurar a largo plazo las condiciones naturales de la producción adquieren creciente importancia. La creación de una ventajosa base nacional demanda del Estado las capacidades necesarias, tanto reguladoras como orientadoras." Mármora y Messner, "Integración", 1991, pp. 165-166.

8 "Los problemas financieros de los pequeños productores pueden abrir el camino a la compra de sus tierras por empresas agrarias de tamaño grande, lo cual refuerza la concentración e incrementa el control extranjero [...] Aún en el caso de que los pequeños productores se logren adaptar, suelen tener que tratar en condiciones desiguales con grandes empresas de exportación, sin gozar de la regulación estatal." Walker, "Ajuste", 1991, p. 18. de trabajo, éste se mantiene y se busca aumentar, principalmente, por el debilitamiento -y aun la desaparición- de las organizaciones de los trabajadores. Para ello se recurre, por ejemplo, al impulso del solidarismo como forma de organización alternativa a los sindicatos y más adecuada a los intereses empresariales. Igual, se recurre a la aplicación de las leyes en contra de las luchas laborales, así como a la cooptación y corporativización o -en Guatemala sobre todo- al uso descarnado de la fuerza.

Por otra parte, la prioridad que le asigna el ajuste a la producçión agropecuaria lleva a la permanencia de las estructuras tradicionales en el campo (ya no más el impulso por parte de la AID de la reforma agraria en El Salvador y Honduras, por ejemplo). Además, se acentúa la dualidad entre sectores "modernos", ligados a las exportaciones, y "atrasados", que producen barato para el consumo local de alimentos y para la demanda de materias primas de algunas de las industrias de punta, manteniendo además bajos los salarios.

A ello se suma una mayor y creciente dependencia del exterior. En este caso es relevante el hecho de que se está acentuando el dominio del agro centroamericano por parte del capital extranjero. Ya no se trata sólo de los enclaves tradicionales (las plantaciones bananeras, por ejemplo) sino que es un dominio que adquiere formas más generales y extendidas. ${ }^{9}$

9 Conviene señalar que, en términos relativos a otros países latinoamericanos, con la clara excepción de Nicaragua, en Centroamérica el peso de la deuda externa sobre la economía 


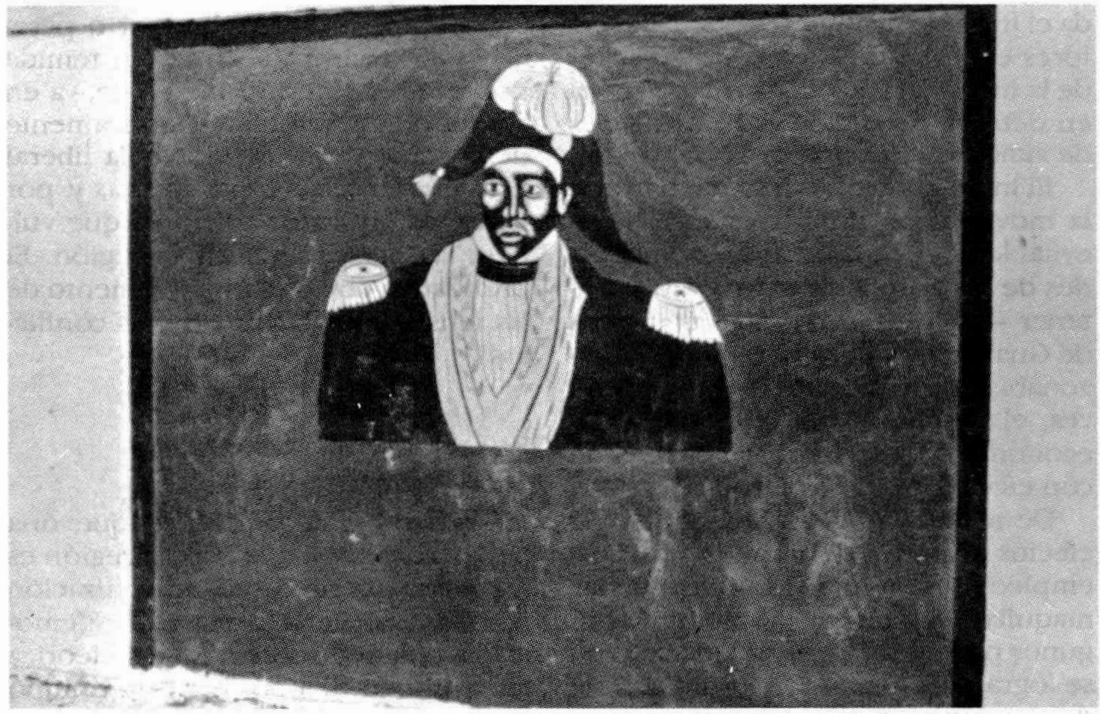

Esta mayor dependencia externa es el resultado, entre otros factores, de la debilidad que presentan las economías centroamericanas ante el exiguo ahorro nacional. Ahora, el mismo es castigado todavía más por el pago del servicio de una deuda que es interminable y que crece con los nuevos préstamos que se hacen "para conseguir dinero fresco". ${ }^{10} \mathrm{~A}$ esto se suman la devaluación constante de la moneda

es bajo. El problema radica en que la misma se encuentra exhausta y que el endeudamiento tiende a crecer, por ejemplo, en relación con las exportaciones de bienes y servicios. Si esa proporción era de $189.8 \%$ en 1981 , para 1989 era de 361\%. Para 1989, los países más afectados en cuanto a esta relación son Nicaragua con $2207 \%$ y Honduras con 306\%. CEPAL, Evolución, 1990 , cuadro 5 , p. 35.

10 Véase, entre otros, Torres Rivas, "Cen- para "ser competitivos", la fuga de capitales, debida a la inestabilidad que no cesa, y las mejores condiciones de ganancia que ofrece el sistema financiero internacional.

Por otra parte, la sustitución de exportaciones exige del acceso y conocimiento de los mercados internacionales, los cuales son privativos o, en todo caso, están dominados por las empresas transnacionales. Además, los países centroamericanos entran a competir con un mercado de productos "no tradicionales" ya saturado y con fluctuaciones de precios y demanda muy grandes y frecuentes. Finalmente, buscando mejorar la "competitividad", se

troamérica", 1989, y para el caso de Guatemala, a Figueroa Ibarra, Recurso, 1991. 
da el traslado de ingresos hacia los sectores exportadores vía la devaluación de la moneda. Esto, como es lógico, va en detrimento de los ya bajos niveles de vida de la mayoria de la población.

$\mathrm{El}$ incremento del descontento y de la movilización populares son así inevitables. Igual, las mayores dificulta. des de los grupos de poder para mantener -0 alcanzar en casos como los de Guatemala y El Salvador-grados razonables de estabilidad. Aquí, entonces, el desencuentro entre política y economía adquiere su total dimensión con efectos de largo plazo.

De manera adicional se suman los efectos negativos del aumento del desempleo, pese a que el impulso de las maquiladoras ofrece de momento algunos paliativos. En efecto, no sólo no se logra abatir con ellas los índices de desempleo sino que, además, se trata de empresas que se basan en los bajos salarios, la evasión-legalizada muchas veces-de las conquistas laborales $y$ de las cargas fiscales y, sobre todo, son industrias de "pies ligeros", prontas a trasladarse a donde les ofrezcan nuevas y mayores "ventajas comparativas". Esto, desde luego, coloca en competencia abierta entre sí a los países más pobres.

Así, el ajuste está contribuyendo a un ensanchamiento todavía mayor de la brecha entre pobres y ricos. Ello ocurre a pesar de los Fondos de Inversión Social (FIS), que se impulsan dentro del ajuste. Éstos están dirigidos a paliar los peores efectos de la crisis y de las políticas neoliberales sobre los más pobres, con lo que la tendencia es a una reducción todavía mayor de las ya exiguas y debilitadas capas medias de la población, condenadas a una lu. cha sin cuartel por sobrevivir o pere. cer, vale decir, caer en la tan temida pobreza. Esto, indudablemente, va en contra de los valores intrínsecamente democráticos que la ideología liberal le asigna a dichas capas medias y, por tanto, es un factor adicional que vulnera las perspectivas de la región. El pronóstico obvio es un incremento de las tensiones sociales y de los conflictos.

\section{UNA PERSPECTIVA PROBABLE}

En general, puede decirse que una perspectiva probable para la región es hacia una suerte de "democratización por vía autoritaria"; ésta es la "democracia posible" sobre la que se teoriza en el último tiempo. Por este camino estaremos asistiendo a renovados intentos, dentro de los grupos de poder, por lograr la "centaurización" del Estado, es decir, convertirlo en un ente capaz de aplicar con alguna ventaja y coherencia la fuerza y, a la vez, impulsar mecanismos que busquen el consenso.

Por eso, ahora en Centroamérica dominan los intentos estatales para lograr el "pacto social", así como el impulso de "elecciones libres y verificables". Pero ello se hace en muchos casos a partir de la exclusión o el debilitamiento previos de los opositores $o$, en situaciones extremas, mediante la eliminación de los mismos de la escena política vía el acoso y la intimidación o la desaparición, el asesinato o el exilio. Los casos de Guatemala y El Salvador son muy claros en este respecto.

En estos dos países, la perspec- 
tiva es un periodo relativamente largo y tortuoso de precaria liberalización; respecto a ellos es difícil prever una transición democrática, en sentido estricto, en el corto plazo. De hecho, los actuales procesos políticos, básicamente de apertura electoral, se dan bajo tutela militar. Esto ocurre en grado menor en Honduras, en tanto que en Costa Rica se comprueba un cierto debilitamiento de la pluralidad política lograda en 1948. Finalmente, en Nicaragua, la moneda aún está en el aire, si bien pareciera ser que la tendencia es hacia la consolidación de los avances democráticos alcanzados a partir de 1979. No obstante, la reactivación de la "Contra" y la persistencia de agudos problemas económicos son signos preocupantes.

En todo caso, una cuestión parece clara: la polarización social en la región tiende a ser cada vez más aguda y la experiencia nos dice que esto sólo traerá nuevos conflictos. Si éstos habrán de traducirse en nuevos ascensos revolucionarios o no, es difícil de decir. Los ascensos de este tipo ocurridos en las décadas pasadas estuvieron sustentados, en buena medida, en las contradicciones gestadas por el tipo de integración económica impulsada por el Mercado Común Centroamericano en los años 60 y 70 . El problema ahora es, sin embargo, más grave pues las nuevas contradicciones se asientan en una economía con serias dificultades de crecimiento.

En resumen: es claro que la democratización pasa por elecciones libres y verificables pero en Centroamérica se necesita, además de un marco propicio para la organización y movilización libres de los opositores y, en general, de la sociedad civil, que los centroa. mericanos en general accedan efectivamente a la condición de ciudadanos, la cual difícilmente se logra sumiéndolos en la miseria. Pero, sobre todo, tal como lo constata la historia reciente, se requiere de una concepción del desarrollo con sentido económico y social, es decir, un crecimiento con equidad, ambientalmente sustentable y en democracia, tal como lo viene señalando últimamente la CEPAL.

En lo inmediato, urge superar la pobreza crítica. Ello demanda, sobre todo, de una reforma agraria concebida más allá de la sola satisfacción de las necesidades de sobrevivencia de la familia campesina. Se necesita Je una reforma agraria articulada en torno a objetivos nacionales, entre otros, relacionados con la seguridad alimentaria. Sobre esta base podría pensarse en agroindustrias y en el impulso de exportaciones no tradicionales que respondan a los volúmenes de divisas que sean realmente necesarios para el desarrollo económico-social. Desde luego, se requiere también de una reforma tributaria que supere el actual carácter regresivo de la estructura fiscal. Así también, de una integración regional democrática (desde dentro y hacia adentro como diría Xabier Gorostiaga) y, desde luego, de un real y efectivo ejercicio de la soberanía.

Éstas son, en definitiva, algunas líneas básicas de trabajo que la propia lucha de los centroamericanos y la reflexión sobre ellas han hecho evidentes. El reto fundamental para estos pueblos es ganar capacidad para desarrollar e impulsar estas líneas. Los 
caminos a seguir dependen de muchas circunstancias y condiciones propias de cada país, pero es evidente que un factor clave es el logro de la democracia, y ésta pasa, como hemos visto, por el desmontaje de los viejos sistemas de acumulación y dominación.

\section{BiBliografía}

-Barrantes, Trino: "El bipartidismo como contexto del proceso electoral costarricense", Secuencia, núm. 17, mayo-agosto de 1990 , pp. 51-70.

-CEPAL, Evolución de la economía centroamericana, 1989, México, 1990.

- Centroamérica: notas sobre la situación de la pobreza y politicas sociales, México, 1991 (offset).

-Figueroa Ibarra, Carlos, El recurso del miedo. Ensayo sobre el Estado y el cerror en Guatemala, EDUCA, Costa Rica, mayo de 1991. .

-Mármora, Leopoldo, y Dirk Messner, "La integración de Argentina, Brasil y
Uruguay: concepciones, objetivos, resultados", Comercio Exterior, vol. XLI, nún. 2, febrero 1991.

-Mayorga Quirós, Román, "El crecimiento desigual en Centroamérica, 1950-2000", tesis, El Colegio de México, México, 1983.

-Morales G., Abelardo, "Costa Rica: caracazo en miniatura", Revista Alai, año, xv, $2^{a}$ época, núm. 142, agosto 1991.

-Peña, Artemio, Ajuste y estrategia productiva. El caso de Guatemala, Centro de Estudios de la Realidad Guatemalteca (CERG), Perspectiva Centroamericana núms. 10-11, septiembre-diciembre 1991 (Colección Temas de la Realidad Guatemalteca).

-Torres Rivas, Edelberto, "Centroamérica: la transición autoritaria hacia ta democracia", en Lorenzo Meyer y José Luis Reyna (coords.), Los sistemas políticos de América Latina, Universidad de las Naciones Unidas/Siglo XXI Editores, México, 1989.

-Walker, Ian, El ajuste estructural y el futuro desarrollo de la región centroame. ricana, Universidad Nacional Autónoma de Honduras, Documento de Trabajo núm. 2, marzo de 1991. 